\title{
Evidence for an inflationary phase transition from the LSS and CMB anisotropy data
}

\author{
J.Barriga ${ }^{a}$ E. Gaztañaga ${ }^{a, b}$, M.G. Santos ${ }^{c}$ and S. Sarkar ${ }^{c}$ \\ anstitut d'Estudis Espacials de Catalunya, IEEC, Institut de Ciències de l'Espai/CSIC, \\ Edf. Nexus-201 - c/ Gran Capità 2-4, 08034 Barcelona, Spain. \\ b INAOE, Astrofísica, \\ Tonantzintla, Apdo. Postal 216 y 51, Puebla 7200, Mexico. \\ ${ }^{\mathrm{c}}$ Theoretical Physics, University of Oxford, \\ 1 Keble Road, OX1 3NP, UK
}

In the light of the recent Boomerang and Maxima observations of the CMB which show an anomalously low second acoustic peak, we reexamine the prediction by Adams et al (1997) that this would be the consequence of a 'step' in the primordial spectrum induced by a spontaneous symmetry breaking phase transition during primordial inflation. We demonstrate that a deviation from scale-invariance around $k \sim 0.1 h \mathrm{Mpc}^{-1}$ can simultaneously explain both the feature identified earlier in the APM galaxy power spectrum as well the recent CMB anisotropy data, with a baryon density consistent with the BBN value. Such a break also allows a good fit to the data on cluster abundances even for a critical density matter-dominated universe with zero cosmological constant.

\section{Introduction}

It is commonly assumed by astronomers that inflation predicts a scale-invariant 'HarrisonZeldovich' (H-Z) spectrum of scalar density perturbations, $P_{0}(k) \propto k^{n}$ with $n=1$. Adams et al. (1997) realized that the spectrum may not be even scale-free because the rapid cooling of the universe during primordial inflation can result in spontaneous symmetry breaking phase transitions which may interrupt the slow roll of the inflaton field for brief periods. This is in fact inevitable in models based on $N=1$ supergravity, the phenomenologically successful extension of the Standard Model of particle physics and the effective field theory below the Planck scale. During inflation, the large vacuum energy breaks (global) supersymmetry giving otherwise massless fields ('flat directions') a mass of order the Hubble parameter [7,6], causing them to evolve rapidly to the asymmetric global minima of their scalar potential. Such 'intermediate scale' fields are generic in models derived from superstring/M-theory and have gauge and Yukawa couplings to the thermal plasma so are initially confined at the symmetric maxima of their scalar potentials. Consequently it takes a (calculable) finite amount of cooling before the thermal barrier disappears and they are free to evolve to their minima [13]. When a symmetry breaking transition occurs, the mass of the inflaton field changes suddenly (through couplings in the Kähler potential), temporarily violating the slow-roll conditions and interrupting inflation (Adams et al. 1997) . Thus the density perturbation is expected to have a (near) $\mathrm{H}-\mathrm{Z}$ spectrum for the first $\sim 10$ e-folds of expansion followed by one or more sudden departures from scale-invariance lasting $\sim 1$ e-fold. In order for such spectral features to be observable in the LSS or CMB, it is of course necessary that they occur within the last $\sim 50$ e-folds of inflation, corresponding to spatial scales going up to the present Hubble radius $H_{0}^{-1} \sim 3000 h^{-1} \mathrm{Mpc}$. Since the density pertur-

\footnotetext{
${ }^{1}$ When (re)heating occurs at the end of inflation such fields may again be forced back to the symmetric maximum, undergoing symmetry breaking a second time when the universe cools down to the electroweak scale in the radiationdominated era and driving a late phase of 'thermal inflation' [9].
} 
bation can be observed on scales from the Hubble radius down to $\sim 1 h^{-1} \mathrm{Mpc}$, corresponding to about 8 e-folds of expansion, it would be not unreasonable to expect at least one such spectral break to be seen today.

\section{Reconstructing the primordial spec- trum}

\subsection{Primordial spectrum from the APM}

There are several arguments (reviewed in the Appendix of [1]) that on scales $0.01 h^{-1} \mathrm{Mpc} \lesssim$ $k \lesssim 0.6 \mathrm{hMpc}^{-1}$, which are at most weakly non-linear, the APM galaxy power spectrum $P_{\mathrm{APM}}(k)$ [4] is an unbiased (or moderately linearly biased) tracer of the mass. The linear power spectrum recovered under this assumption from $P_{\mathrm{APM}}(k)$ is well fitted in this range by $\left[5: P_{\operatorname{lin}}(k) \simeq \frac{7 \times 10^{5} k\left(h^{-1} \mathrm{Mpc}\right)^{3}}{\left[1+\left(k / k_{c}\right)^{2}\right]^{1.6}}\right.$ where $k_{\mathrm{c}}=$ $150\left(H_{0} / c\right) \simeq 0.05 \mathrm{~h} \mathrm{Mpc}^{-1}$. Here we will also use the common convention: $P_{\text {lin }}(k) \equiv P^{0}(k) T^{2}(k)$ where $P^{0}(k)$ is the primordial spectrum of matter fluctuations and $A$ is the (dimensionful) normalisation constant. From the expressions above we estimate $P^{0}(k)$ to be :

$P^{0}(k)= \begin{cases}A_{1} k, & k<k_{1}, \\ A k \frac{1 / T_{\mathrm{CDM}}^{2}(k)}{\left[1+\left(k / k_{c}\right)^{2}\right]^{1.6}}, & k_{1} \leq k \leq k_{2}, \\ A_{2} k, & k_{2}<k,\end{cases}$

$T_{\mathrm{CDM}}=\left[1+a k+(b k)^{3 / 2}+(c k)^{2^{\nu}}\right]^{-1 / \nu}$ given by [?], where $a=6.4 \Gamma^{-1} h^{-1} \mathrm{Mpc}, b=3 \Gamma^{-1} h^{-1} \mathrm{Mpc}$, $c=1.7 \Gamma^{-1} h^{-1} \mathrm{Mpc}, \nu=1.13$ and the 'shape parameter' $\Gamma=\Omega_{\mathrm{m}} h \mathrm{e}^{-\left[\Omega_{\mathrm{B}}\left(1+\sqrt{2 h} / \Omega_{\mathrm{m}}\right)-0.06\right]} . \quad A=$ $7 \times 10^{5}\left(h^{-1} \mathrm{Mpc}\right)^{3}$ and $A_{1}$ and $A_{2}$ are such that make $P^{0}(k)$ continuous. For the cosmological parameters which define $\Gamma$ we consider the observationally indicated values $h \sim 0.5-0.8$ and $\Omega_{\mathrm{B}}=\left(0.019_{-0.0012}^{+0.0013}\right) h^{-2}$ [8] (for further discussion see [1)). Figure 1 shows the recovered primordial spectrum (1) for two choices of $\Gamma$ corresponding to the sCDM model $(\Gamma=0.5)$ and a low density variant. Note that the $\Gamma=0.5$ reconstruction has significantly less power than a scale-free $\mathrm{H}$ $\mathrm{Z}$ spectrum on scales $k \gtrsim 0.1 \mathrm{hMpc}^{-1}$, while the $\Gamma=0.2$ reconstruction is closer to a $\mathrm{H}-\mathrm{Z}$ spectrum but has relatively more power. Thus the latter possibility does not give a good fit to the
Boomerang/MAXIMA data with the value (given above) of the baryon density from big bang nucleosynthesis (the reader is referred to [1] for further comments on this).

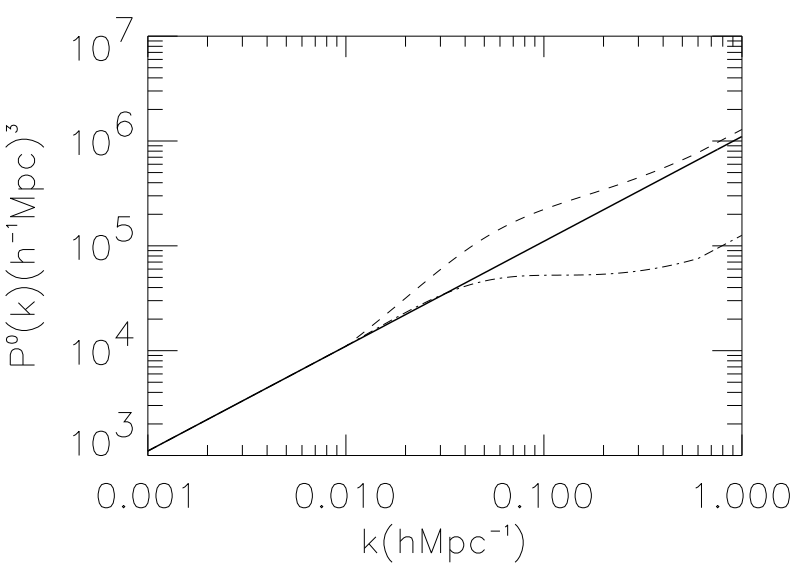

Figure 1. Reconstruction of the primordial density power spectrum from the APM data, adopting a CDM shape parameter $\Gamma$ of 0.5 (dot-dashed line) and 0.2 (dashed line). A Harrison-Zeldovich spectrum (full line) is shown for comparison.

\subsection{Fits to the CMB and LSS data}

We parameterise the "step" in the primordial power spectrum (see Figure 1) as:

$P^{0}(k)= \begin{cases}A k, & k \leq k_{\text {start }} \\ C k^{\alpha}, & k_{\text {end }} \leq k \leq k_{\text {start }} \\ B k, & k \geq k_{\text {end }}\end{cases}$

where $k_{\text {start }}$ and $k_{\text {end }}$ mark the begining and end of the break from $\mathrm{H}-\mathrm{Z}$ spectra with amplitudes $A$ and $B$. (The values of $C$ and $\alpha$ are specified by the other parameters.) In the multiple inflation model [2], the actual form of the spectrum during the phase transition is difficult to calculate since the usual 'slow-roll' conditions are violated. However a robust expectation is that $\ln \left(k_{\text {end }} / k_{\text {start }}\right) \sim 1$ because the field undergoing the symmetry-breaking phase transition evolves 


\begin{tabular}{|l|l|l|l|l|l|}
\hline$\Omega_{\Lambda}$ & $h$ & $k_{\text {start }}$ & $A / B$ & $\sigma_{8}$ & $\chi^{2}$ \\
\hline \hline 0.0 & 0.50 & 0.07 & $4.3-6.5$ & $0.65-0.73$ & 11.9 \\
\hline 0.2 & 0.55 & 0.06 & $3.6-5.6$ & $0.69-0.78$ & 10.0 \\
\hline 0.3 & 0.60 & 0.06 & $3.4-5.0$ & $0.76-0.85$ & 10.3 \\
\hline 0.4 & 0.65 & 0.05 & $3.1-4.6$ & $0.77-0.87$ & 13.1 \\
\hline 0.5 & 0.70 & 0.05 & $2.6-4.1$ & $0.81-0.93$ & 18.8 \\
\hline 0.6 & 0.75 & 0.05 & $2.0-2.8$ & $0.90-0.99$ & 29.7 \\
\hline
\end{tabular}

Table 1

Parameters for best fits to $\mathrm{CMB}+\mathrm{APM}$ data with the BBN constraint on the baryon density.

exponentially fast to its minimum. The ratio of the amplitudes $A / B$ is determined by the (unknown) superpotential couplings of the field undergoing the phase transition but is expected to exceed unity (i.e. there is a decrease in the power). Table 1 shows the result of imposing the BBN constraint and the Hubble parameter range mentioned in the subsection before and also requiring that $0.5 \leq \ln \left(k_{\text {end }} / k_{\text {start }}\right) \leq 2$. We see that the data now prefer lower values of $\Omega_{\Lambda}$ (and $h$ ).In particular the value $\Omega_{\Lambda} \sim 0.7$ favoured by the SN Ia data 10, 12 is not permitted. Figure 2 shows the fit to the CMB data for these models.

\subsection{Implications for cluster abundances}

Table 1 shows the value of the variance $\sigma(R)$ in (dark matter) fluctuations (normalised to the $\mathrm{CMB}$ ), over a sphere of size $R=8 h^{-1} \mathrm{Mpc}$ : $\sigma^{2}(R)=1 /\left(2 \pi^{2}\right) \int_{0}^{\infty} W^{2}(k R) P_{l i n}(k) T^{2}(k) k^{2} \mathrm{~d} k$ using a 'top hat' smoothing function, $W(k R)=$ $3\left[\frac{\sin (k R)}{(k R)^{3}}-\frac{\cos (k R)}{(k R)^{2}}\right]$. The tabulated values are smaller than the values we would obtain when using a $\mathrm{H}-\mathrm{Z}$ primordial spectrum, since the $\Gamma$ values for these cosmological models are quite high which, according to the APM data (see Figure 1), implies a decrease power for the relevant scales. In Figure 3 we show the Press-Schechter predictions for the redshift evolution of cluster number densities compared to the observations, as presented in Bahcall \& Fan (1998). Here we are displaying the predictions for clusters with $M>8 \times 10^{14}$ solar masses. The flat H-Z $\Omega_{\Lambda}=0.2$ (ie $\Omega_{m} \simeq 0.8$ ) model normalisedd to COBE (top thick continuous line) fails to match the observations of cluster abundances, a fact that has been

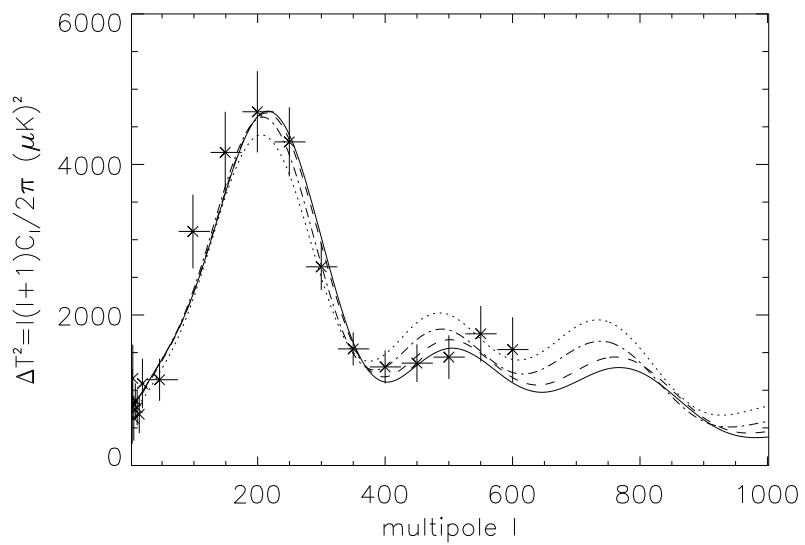

Figure 2. CMB angular power spectra for the bestfit models in Table 1 with $\Omega_{\Lambda}=0$ (continuous line), $\Omega_{\Lambda}=0.2$ (dashed line), $\Omega_{\Lambda}=0.4$ (dot-dashed line), and $\Omega_{\Lambda}=0.6$ (dotted line). All models have the BBN baryon density $\Omega_{\mathrm{B}}=0.019 / h^{2}$. The data shown are from COBE and Boomerang.

used to rule out this model. The figure shows how the models fitted in Table1 1 do match the redshift evolution, specially after allowing for the possibility mentioned above of having a slightly smaller $\sigma_{8}$ values to better match the $z=0$ data. Note that these predictions have not been adjusted in any way to predict cluster abundances, all parameters were fixed by the APM-CMB fit.

\section{Discussion}

Allowing the primordial spectrum not to be scale-invariant has interesting consequences. In our analysis we find that high values of $\Omega_{m}$ in a flat universe (and therefore low values of $\Omega_{\Lambda}$ ) are compatible with CMB and LSS data. The standard interpretation of LSS data (eg APM $P(k)$ and cluster abundances) favours of a low $\Gamma \sim \Omega_{m} h \sim 0.2$ for a $\mathrm{H}-\mathrm{Z}$ spectrum. But these observations can also be explained with larger values of $\Omega_{m}$ if we allow for a break in the primordial spectrum. Our best fit values in Table 1 imposing the BBN constraint mentioned above and the Hubble parameter constraint in range, prefer 


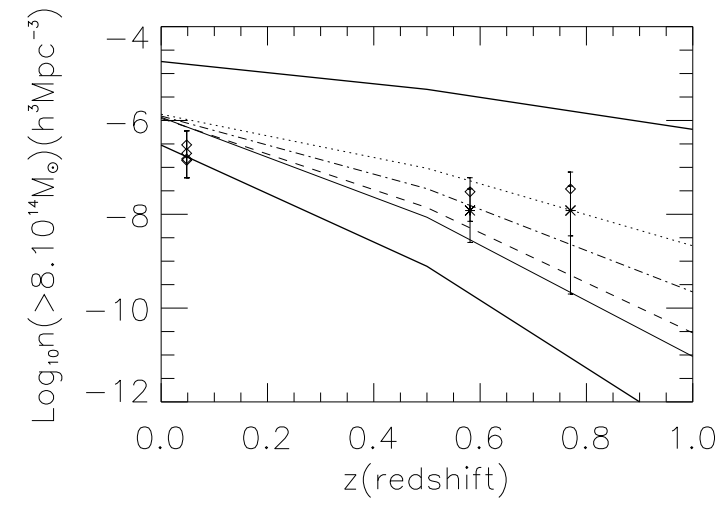

Figure 3. Number density of massive clusters with $M>8 \times 10^{14}$ solar masses as a function of redshift. Points with errorbars correspond to the observations as depicted in Bahcall \& Fan 1998. The thick continuous lines correspond to the P-S prediction for the flat $\mathrm{H}-\mathrm{Z}$ model with $\Omega_{\Lambda}=0.2$ (ie $\Omega_{m} \simeq 0.8$ ), normalised to COBE (top line) and scaled to match the cluster abundance at $z=0$ (bottom line). The middle lines correspond to the best fitted models in Table 1 with $\Omega_{\Lambda}=0$ (continuous line), $\Omega_{\Lambda}=0.2$ (dashed line), $\Omega_{\Lambda}=0.4$ (dot-dashed line), and $\Omega_{\Lambda}=0.6$ (dotted line).

higher values of $\Omega_{m}$ (and lower values of $\Omega_{\Lambda}$ ). The required spectral break decreases with increasing $\Omega_{\Lambda}$ but one cannot do without such a break. The value $\Omega_{\Lambda} \sim 0.7$ favoured by the SN Ia data 10,12 is not permitted, while it is possible to have a universe with no cosmological constant and $\Omega_{m} \simeq 1$.

The hypothesis of a primordial density perturbation with broken scale-invariance is eminently falsifiable. The ongoing $2 \mathrm{DF}$ and SDSS redshift surveys can confirm or rule out such a feature in the power spectrum of galaxy clustering, while the forthcoming MAP mission will determine whether all the secondary acoustic peaks in the CMB angular spectrum are indeed suppressed as expected. Broken scale-invariance has a natural explanation in a phase transition occuring during inflation as expected in supersymmetric theories. If established this would provide the first direct connection between astronomical data and physics at very high energies. (The reader is referred to [1] for further details, references and acknowledgements).

\section{Acknowledgments}

J.B. would like to thank INAOE for their warm hospitality. J.B. and E.G. acknowledge support by grants from IEEC/CSIC and DGES(MEC)(Spain) project PB96-0925, and Acción Especial ESP1998-1803-E. The work of M.G.S. was supported by the Fundação para a Ciencia e a Tecnologia under program PRAXIS XXI/BD/18305/98.

\section{REFERENCES}

1. Barriga J., Gaztañaga E., Santos M., Sarkar S. astro-ph/0011398.

2. Adams J.A., Ross G.G., Sarkar S., 1997, Nucl. Phys., B503, 405, hep-ph/9704286.

3. Bahcall N., Fan, X., 1998, ApJ, 504, 1.

4. Baugh C.M., Efstathiou G.P., 1993, MNRAS, 265, 145.

5. Baugh C.M., Gaztañaga E., 1996, MNRAS, 280, L37.

6. Coughlan G., Fischler W., Kolb E.W., Raby S., Ross G.G., 1984, Phys. Lett., 140B, 44.

7. Dine M., Fischler W., Nemechansky D., 1984, Phys. Lett., 136B, 169.

8. Fiorentini G., Lisi E., Sarkar S., Villante F., 1998, Phys. Rev., D58, 063506.

9. Lyth D., Stewart E.D., 1996, Phys. Rev., D53, 1784.

10. Perlmutter S. et al., 1999, ApJ, 517, 565.

11. Press, W.H., Schechter, P., 1974, ApJ 187, 425.

12. Riess A.G. et al., 1998, AJ, 116, 1009.

13. Yamamoto K., 1986, Phys. Lett., 168B, 341. 